\title{
35
}

\section{Imaging actin dynamics throughout ingression membrane system development}

Tom O'Sullivan $^{1,2}{ }^{\text {, Steve Thomas }}{ }^{1}$, Martyn Mahaut-Smith ${ }^{2}$

$1_{\text {Institute of Cardiovascular Sciences, College of Medical and Dental }}$ Sciences, University of Birmingham., United Kingdom. ${ }^{2}$ Department of Molecular and Cellular Biology, University of Leicester, United Kingdom

\section{Abstract Text}

Platelets are essential blood cells required for haemostasis. Both acquired and inherited conditions can give rise to either a reduced platelet count, known as thrombocytopenia, or can lead to platelet function disorders. Therefore, understanding how platelets are produced from their progenitor cells, megakaryocytes, is critical in understanding these cells in health and disease.

The invaginated membrane system (IMS) is an internalised reservoir of lipid membrane within late stage megakaryocytes that provides cell membrane mass for the production of future platelets (Machlus \& Italiano, 2013). IMS development and maturation is intimately linked to actin cytoskeleton function, with dysregulation of the actin cytoskeleton leading to defects in IMS maturation (Antkowiak et al., 2016). However, the relationship between actin cytoskeleton and IMS is not fully understood, and many of the key regulatory proteins are yet to be identified.

The primary aim of this work is to quantitatively describe the stages of actin organisation during IMS development by use of Dual inverted Single Plane Illumination Microscopy (DiSPIM), Lifeact-GFP megakaryocytes and plasma membrane dyes. By monitoring actin and membrane dynamics in 3D for up to $12 \mathrm{hrs}$, and subsequent tracking and image analysis of cells at different stages of maturation, we are able to generate a range of quantitative descriptors of the IMS. This allows us to build a model of normal IMS 
development and to investigate how disruption to the cytoskeleton perturbs this. Ultimately, this will allow us to understand how genes which regulate the cytoskeletal, can lead to thrombocytopenia when affected. 